Research paper

\title{
Influence of passage number on the impact of the secretome of adipose tissue stem cells on neural survival, neurodifferentiation and axonal growth
}

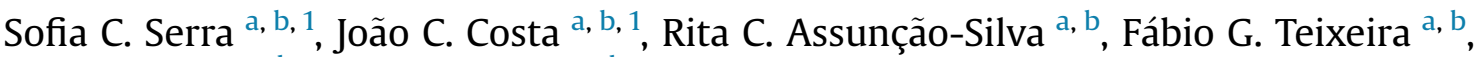

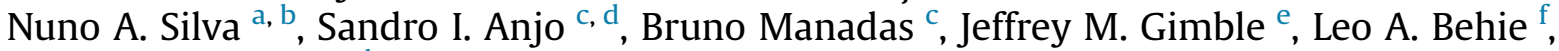 \\ António J. Salgado ${ }^{\text {a, b, * }}$ \\ a Life and Health Sciences Research Institute (ICVS), School of Medicine, University of Minho, Braga, Portugal \\ b ICVS/3B's - PT Government Associated Laboratory, Braga/Guimarães, Portugal \\ ${ }^{c}$ CNC - Center for Neuroscience and Cell Biology, University of Coimbra, Portugal \\ ${ }^{\mathrm{d}}$ Faculty of Sciences and Technology, University of Coimbra, Portugal \\ e Centre for Stem Cell Research and Regenerative Medicine, Tulane University, New Orleans, USA \\ ${ }^{\mathrm{f}}$ Pharmaceutical Production Research Facility (PPRF), Schulich School of Engineering, University of Calgary, Calgary, Canada
}

\section{A R T I C L E I N F O}

\section{Article history:}

Received 10 April 2018

Received in revised form

13 September 2018

Accepted 20 September 2018

Available online $\mathrm{xxx}$

\section{Keywords:}

Secretome

Adipose tissue stem cells

Central nervous system

Differentiation

Axonal growth

Proteomics

\begin{abstract}
A B S T R A C T
Mesenchymal stem cells (MSCs), and within them adipose tissue derived stem cells (ASCs), have been shown to have therapeutic effects on central nervous system (CNS) cell populations. Such effects have been mostly attributed to soluble factors, as well as vesicles, present in their secretome. Yet, little is known about the impact that MSC passaging might have in the secretion therapeutic profile. Our aim was to show how human ASCs (hASCs) passage number influences the effect of their secretome in neuronal survival, differentiation and axonal growth. For this purpose, post-natal rat hippocampal primary cultures, human neural progenitor cell (hNPCs) cultures and dorsal root ganglia (DRGs) explants were incubated with secretome, collected as conditioned media (CM), obtained from hASCs in P3, P6, P9 and P12. Results showed no differences when comparing percentages of MAP-2 positive cells (a mature neuronal marker) in neuronal cultures or hNPCs, after incubation with hASCs secretome from different passages. The same was observed regarding DRG neurite outgrowth. In order to characterize the secretomes obtained from different passages, a proteomic analysis was performed, revealing that its composition did not vary significantly with passage number P3 to P12. Results allowed us to identify several key proteins, such as pigment epithelium derived factor (PEDF), DJ-1, interleucin-6 (IL-6) and galectin, all of which have already proven to play neuroprotective and neurodifferentiating roles. Proteins that promote neurite outgrowth were also found present, such as semaphorin 7A and glypican-1. We conclude that cellular passaging does not influence significantly hASCs's secretome properties especially their ability to support post-natal neuronal survival, induce neurodifferentiation and promote axonal growth.
\end{abstract}

() 2018 Published by Elsevier B.V.

\section{Introduction}

Mesenchymal stem cells (MSCs) were isolated for the first time by Friedenstein and colleagues in 1974 [1]. Classified as a subgroup

\footnotetext{
* Corresponding author. ICVS, School of Medicine, University of Minho, 4710 057Braga, Portugal.

E-mail address: asalgado@med.uminho.pt (A.J. Salgado).

1 Equally contributing authors.
}

of non-hematopoietic multipotent adult stem cells, they have the ability for self-renewal and differentiation in multiple cell lines $[2,3]$. It is possible to isolate them from almost all tissues, but the most common sources for MSCs are bone marrow (BM-MSCs), adipose tissue (ASCs) and the Wharton jelly of the umbilical cord (WJSCs and HUCPVCs) [1,4-7].

In the scope of regenerative medicine, and specially of cellbased therapies, the growing interest for potential applications of MSCs is due to their broad distribution and easy access, huge 
proliferative potential and immunomodulation properties $[3,8]$.

Several studies have emerged where application of MSCs is used in Central Nervous System (CNS) repair models. Indeed, the transplantation of MSCs has already proven to possess therapeutic effects in animal models of Parkinson's Disease $[9,10]$ and spinal cord injury [11,12]. Nowadays it is well established that it is the secretome of these cells - described as all the soluble factors and vesicles secreted to the extracellular milieu - that acts as the main mediator within CNS and other tissues related therapies [13-16]. Indeed, MSCs ability to promote neuronal survival and neurogenesis has been proven in vitro and attributed to their capability to secrete neurotrophic factors such as BDNF and $\beta$-NGF [17]. Characterization of BM-MSCs secretome, obtained as conditioned medium (CM), demonstrated that they were capable of secreting IGF-1, HGF, VEGF and TGF- $\beta$, molecules responsible for in vitro neuronal survival and neurogenesis [17,18]. Some growth factors were also identified in the secretome of ASCs, such as bFGF, NGF and SCF, along with IGF-1, HGF, VEGF and TGF- $\beta 1$, capable of promoting proliferation of neuronal and glial cells populations $[13,14,19]$. Similar results were described after WJ-MSCs and HUCPVCs secretome analysis $[14,15,20]$. Based on these results, the application of MSCs secretome in CNS regeneration models, instead of cell transplantation, seems to be a truly valid alternative.

In spite the growing amount of studies on MSCs and their secretome in the last years, little is known about how cell passaging might influence the secretome composition. In the regenerative medicine field this becomes even more relevant, since both cell transplantation and secretome application require large numbers of cells, which implies cellular expansion up to the required amount through successive passaging.

A study focusing on how cell passaging impacts on MSCs secretome is long overdue and so the aim of the present work was to evaluate how cell passage impacts the effect of human adipose tissue mesenchymal stem cells (hASCs) secretome at various processes (survival, differentiation and axonal growth) in neuronal populations.

The results allowed us to determine that, regardless of the passage, hASCs secretome seems to promote consistently the same effect on the maintenance of post-natal rat hippocampal primary cultures, neuronal differentiation in human neural progenitor cells (hNPCs) and axonal growth from a dorsal root ganglion (DRG) model. This idea is reinforced by the fact that the expression of several proteins involved in those processes also suffers no change with passage number.

\section{Materials and methods}

\subsection{Human adipose-derived stem cell culture}

Human ASCs were isolated from human lipoaspirates obtained from three consenting donors under an institutional review board approved protocol at LaCell LLC. hASCs from three different donors were kindly provided by Professor Jeffrey Gimble (LaCell LLC, New Orleans, Louisiana, USA). Upon thawing, they were plated at a density of $4.0 \times 10^{3}$ cells $/ \mathrm{cm}^{2}$, they were cultured in $\alpha$-MEM medium (Invitrogen, USA), supplemented with $10 \%$ fetal bovine serum (FBS) (Biochrom AG, Germany) and $1 \%$ penicillin-streptomycin (Invitrogen, USA). Cultures were maintained at $37^{\circ} \mathrm{C}$ and $5 \% \mathrm{CO}_{2}$, with medium changes every 2 or 3 days, until confluent.

\section{2. hASC secretome (conditioned media, CM, collection)}

Cells were plated at a density of $4.0 \times 10^{3}$ cells $/ \mathrm{cm}^{2}$ (for cell culture tests), or $12.0 \times 10^{3}$ cells $/ \mathrm{cm}^{2}$ (for proteomic analysis) and left for $72 \mathrm{~h}$ in $\alpha$-MEM medium, supplemented and incubated the same way as above. Afterwards, medium was removed and cells were washed three times with PBS, no $\mathrm{Ca}^{2+}$ or $\mathrm{Mg}^{2+}$, (Invitrogen, USA) and one time with Neurobasal A medium (Invitrogen, USA), supplemented solely with $1 \%$ Kanamycin (Invitrogen, USA) - or Neurobasal medium (Invitrogen, USA), supplemented with 1\% penicillin-streptomycin, in the case of future use with DRGs. After washing, the same supplemented Neurobasal A - again, or Neurobasal - was added to the cells and left for $24 \mathrm{~h}$, at the same incubation parameters. Following this conditioning period, medium (i.e. the secretome of hASCs) was collected and stored at $-80^{\circ} \mathrm{C}$, after a flash-freeze in liquid nitrogen, for use when needed. This procedure was performed for collecting secretomes in P3, P6, P9 and P12.

\subsection{Post-natal rat hippocampal primary culture and incubation with hASCS-CM}

Upon dissection of P4 Wistar Han brains, hippocampi were submitted to a trypsin-based (Sigma, USA) enzymatic digestion, followed by mechanical dissociation. The cells obtained this way were plated, at a density of $4.0 \times 10^{4}$ cells $/ \mathrm{cm}^{2}$, on coverslips coated with poly-D-lysine (Sigma, USA). Cells were then incubated with the previously collected secretomes for 7 days. At culture day 4, half the volume was replaced.

\subsection{Human neural progenitor cell culture and incubation with hASCS-CM}

hNPCs were kindly provided by Professor Leo A. Behie (University of Calgary, Calgary, Canada). Upon thawing, hNPCs in the form of spheres were cultured in a serum-free medium, PPRFh2 [21]. After 2 days, spheres were harvested and mechanically dissociated into a single cell suspension and cultured again with fresh medium. Every 3 or 4 days, $40 \%$ of the used medium was replaced. After 14-20 days, hNPCs were passaged and plated on a pre-coated - poly-D-lysine and laminin (Sigma, USA) - 24-well plate, at a density of $1.0 \times 10^{5}$ cells per well. The secretomes previously collected, at the day of the experiment further supplemented with $1 \%$ glutamax (Gibco, USA), were added immediately to the cells and left for 5 days, at $37{ }^{\circ} \mathrm{C}$ and $5 \% \mathrm{CO}_{2}$. No medium changes or additions were done during that period of time.

\subsection{DRGs culture and incubation with hASCs-CM}

To ensure DRGs adhesion, a matrix of collagen hydrogel droplets was used. After preparation, collagen droplets were incubated for $2 \mathrm{~h}$, at $37^{\circ} \mathrm{C}$ and $5 \% \mathrm{CO}_{2}$. In the meanwhile, DRGs were isolated from P5-P7 Wistar Han pups and at the end placed on top of the previously prepared collagen droplets. Secretomes from the different passaged hASCs were then immediately added, after further supplementation with B27, L-glutamine and glucose. DRGs were kept in culture for 4 days, at $37^{\circ} \mathrm{C}$ and $5 \% \mathrm{CO}_{2}$, with no medium being replaced or added.

\subsection{Immunocytochemistry}

All cells/explants were fixed using 4\% paraformaldehyde, permeated by incubating with $0.3 \%$ Triton X-100 in PBS and blocked with 10\% FBS in PBS. Following all these steps, incubation with primary antibody was performed. Depending on the culture, different antibodies were used as follows: a) mouse antimicrotubule-associated protein 2 (MAP-2) (Sigma, USA) to detect mature neurons in post-natal rat hippocampal primary cultures and in hNPCs cultures; b) mouse anti-neurofilament (NF) (Millipore, USA) to identify neurite projections in DRGs cultures. After washing with PBS, incubation with secondary antibody followed, 
using Alexa Fluor 594 goat anti-mouse immunoglobulin G (IgG) for MAP-2 and Alexa Fluor 488 goat anti-mouse immunoglobulin G (IgG) for NF. Cell samples obtained this way were observed under a BX-61 fluorescence microscope (Olympus, Germany). For this purpose, three coverslips per condition and ten representative fields were chosen and analyzed. In the case of post-natal rat hippocampal primary cultures, results are shown as percentage of MAP-2 positive cells over the total number of cells per field of observation. On the other hand, for hNPCs cultures results are represented by the percentage of differentiated MAP-2 positive cells over the total number of cells per field of observation. For this purpose differentiated cells were considered to be MAP-2 positive cells disclosing the growth of neurites/axons from their cell bodies. Regarding DRGs explants cultured on collagen hydrogel droplets, they were imaged using a FV1000 confocal microscope (Olympus, Germany) and analyzed with Image J software, in order to quantify the area occupied by the neurites within the gel [22]. For that purpose, image scale was first set and then the area occupied by the DRG itself was excluded. Images were then converted to 8 bits and binary. Through the "analyze particles" menu, the software automatically calculated the area of neurite outgrowth, considering the image dark background as contrast.

\subsection{Untargeted Proteomics: mass spectrometry analysis}

Replicates of secretomes from different donors for each passage of hASCs were processed as previously described [23]. Secreted proteins were precipitated from the concentrated medium using the trichloroacetic acid-acetone procedure [24]. The protein pellets were resuspended in $2 \times$ Laemmli buffer (BioRad), aided by ultrasonication and denaturation at $95^{\circ} \mathrm{C}$. After denaturation, samples were alkylated with acrylamide and subjected in gel digestion by using the short-GeLC approach [25]. The formed peptides were subjected to SPE using OMIX tips with C18 stationary phase (Agilent Technologies, Glostrup, Denmark) before liquid chromatography-tandem mass spectrometry (LC-MS/MS). Samples were analyzed on a Triple TOFTM 5600 System $\left(A B\right.$ Sciex $\left.^{\circledR}\right)$ in two phases: information-dependent acquisition (IDA) for protein identification and SWATH acquisition for protein quantification (detailed information in Supplementary Data). A specific library of precursor masses and fragment ions was created by combining all files from the IDA experiments, and used for subsequent SWATH processing. Libraries were obtained using Protein Pilot ${ }^{\mathrm{TM}}$ software (v4.5, AB Sciex ${ }^{\circledR}$ ) searching against the SwissProt database (released October 2014).

SWATH data processing was performed using SWATH ${ }^{\mathrm{TM}}$ processing plug-in for PeakView ${ }^{\mathrm{TM}}\left(\mathrm{v} 2.0 .01\right.$, ABSciex $^{\circledR}$ ). Briefly, peptides were selected automatically from the library and up to 15 peptides with up to 5 fragment ions were chosen per protein. Quantitation was attempted for all proteins in the library file that were identified below 5\% local FDR from ProteinPilot ${ }^{\mathrm{TM}}$ searches, by extracting the peak areas of the target fragment ions of the identified peptides using an extracted-ion chromatogram (XIC) window of 4 min with 20 mDa XIC width.

All peptides that met the $1 \%$ FDR threshold were retained and the levels of human proteins were estimated by summing all the respective transitions and peptides that met the FDR criteria (an adaptation of [26]). For comparisons between experiments, the protein levels were normalized to the internal standard added after medium collection.

\subsection{Statistical analysis}

All statistical analyses were performed using GraphPad Prism 6 for Windows (GraphPad Software). Differences among groups were assessed by one-way ANOVA. A p-value $<0.05$ (95\% confidence level) was set as the criteria for statistical significance. All data are presented as mean \pm standard deviation (SD).

\section{Results}

The purpose of our study was to determine the impact that cell passaging might have on the secretome of hASCs, more specifically in what way it affected its potential to promote maintenance of post-natal rat hippocampal primary cultures as well as its ability to induce neuronal differentiation in human neural progenitor cells or axonal growth in a DRG model.

To determine the effects of the hASCs secretome, we started by testing secretomes collected after different passages (P3, P6, P9 and $\mathrm{P} 12$ ) in post-natal rat hippocampal primary cultures. After a

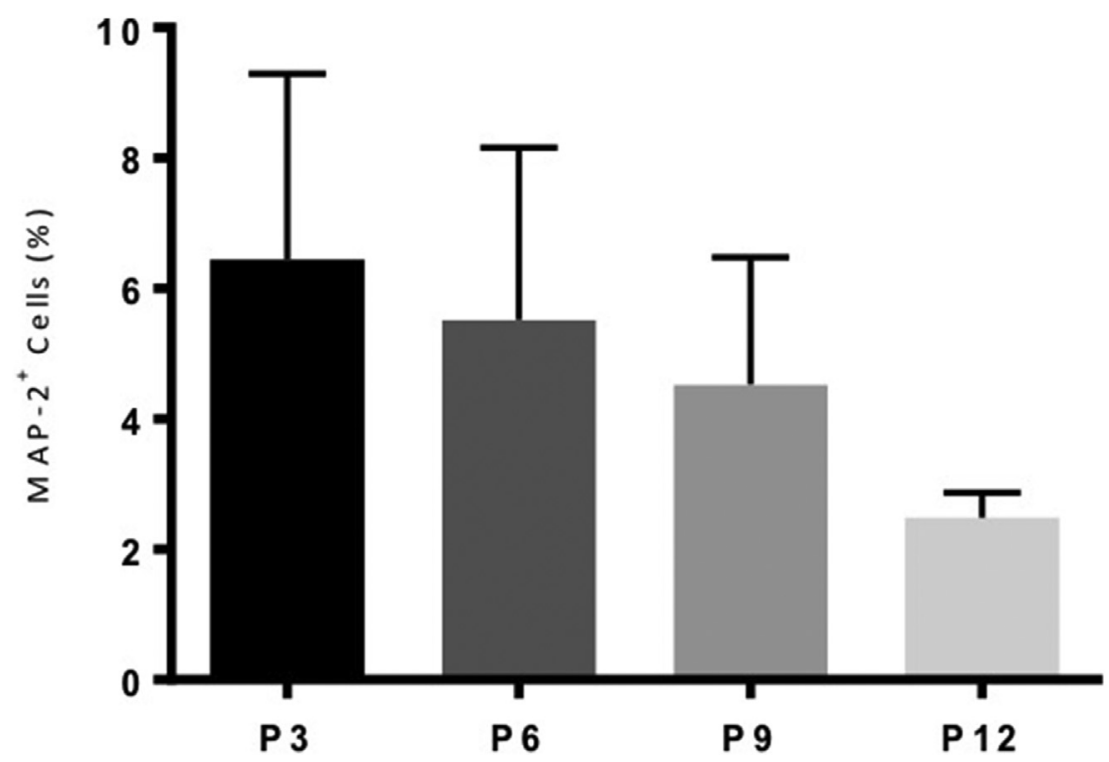

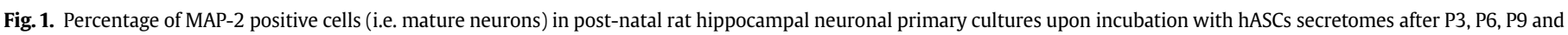
P12. No statistically significant differences found (one-way ANOVA, $n=3$, mean $\pm S D, p<0,05$ ). 

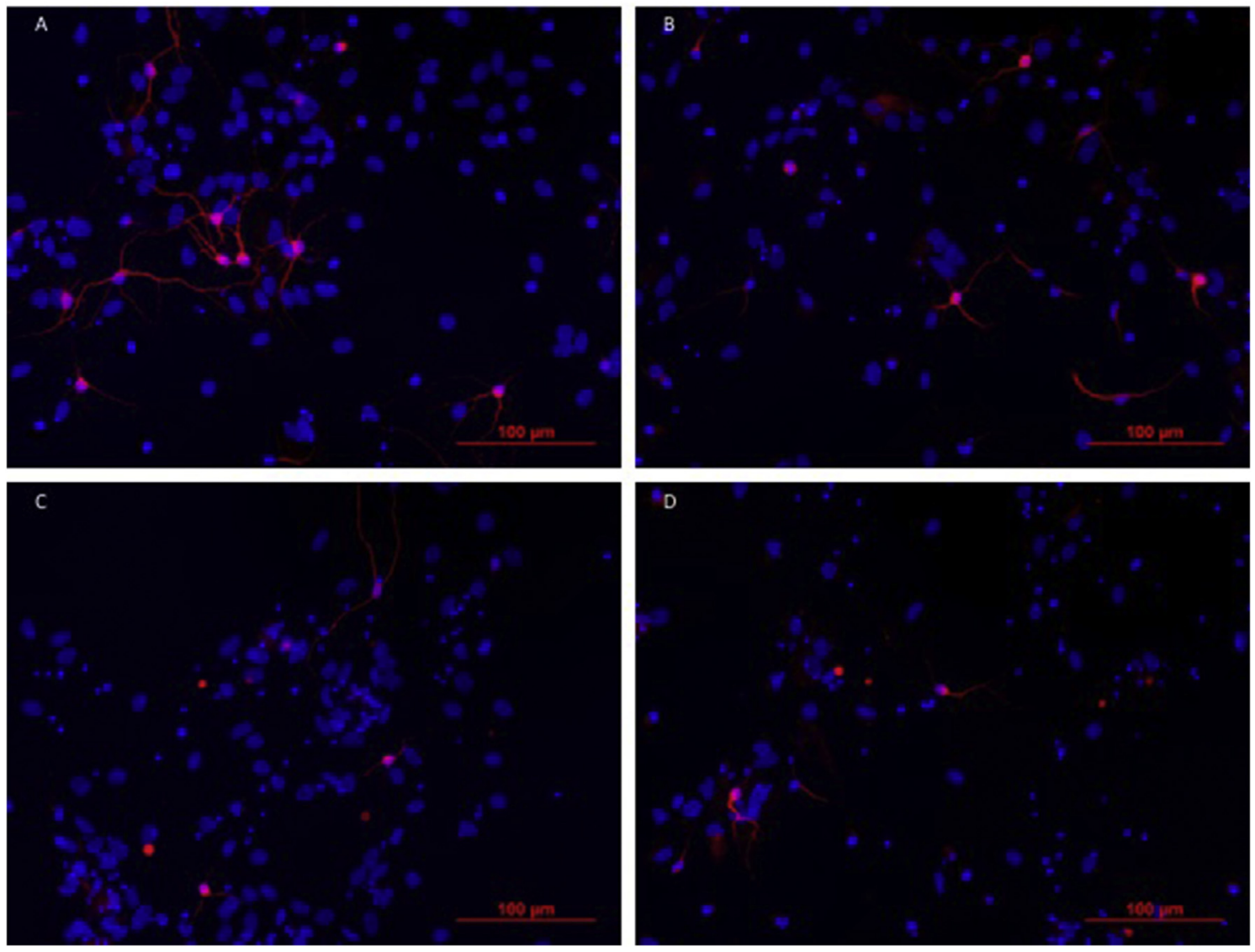

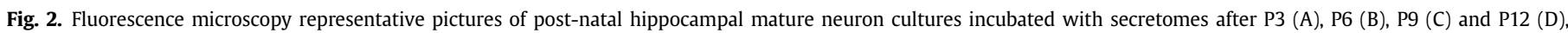
marked for MAP-2 (red) and DAPI (blue, i.e. nucleus marker).

standard culture period, immunocytochemistry for MAP-2 (mature neuronal marker) positive cells was performed. Results revealed that the incubation with the different CM collected did not show statistically significant differences in the percentage of mature neurons (MAP-2 ${ }^{+}$cells) (Figs. 1 and 2 ).
In a similar way, neuronal differentiation was accessed by incubating hNPCs with the collected secretomes. After immunocytochemistry for the same marker, the number of differentiated MAP-2 positive cells was determined. No statistically significant differences were found in the percentage of differentiated cells

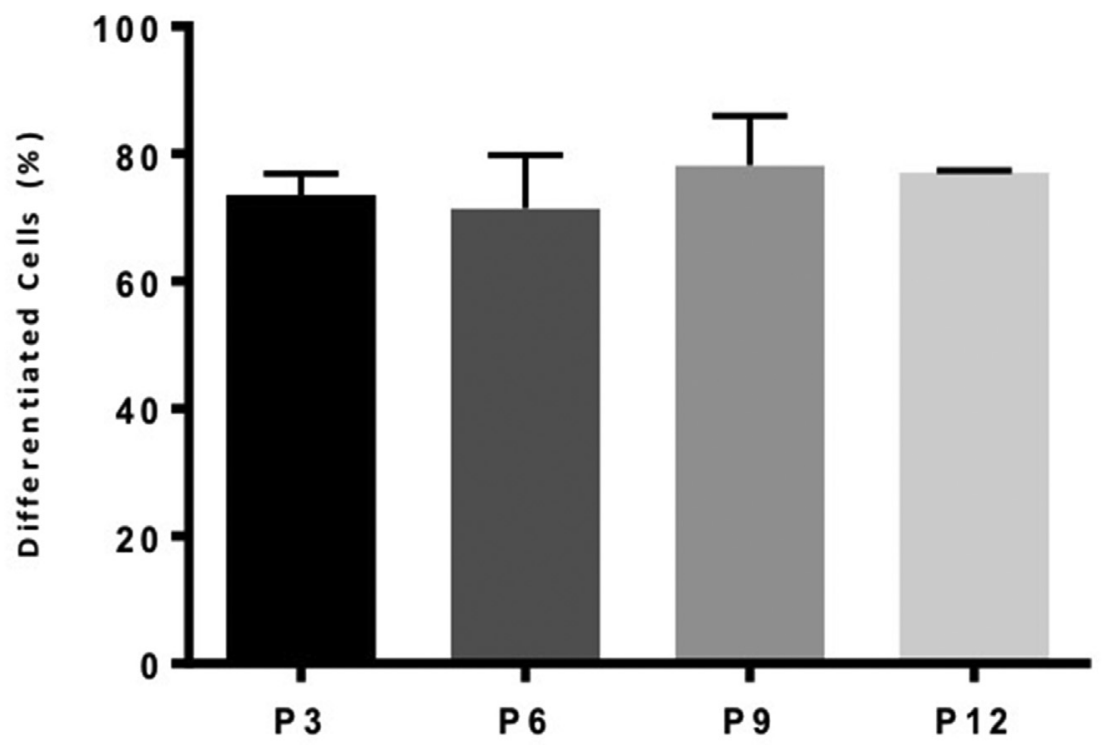

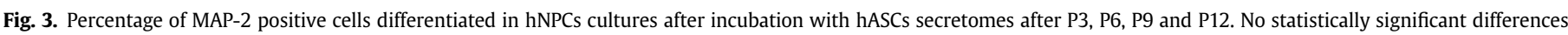
found (one-way ANOVA, $n=3$, mean $\pm S D, p<0,05$ ). 

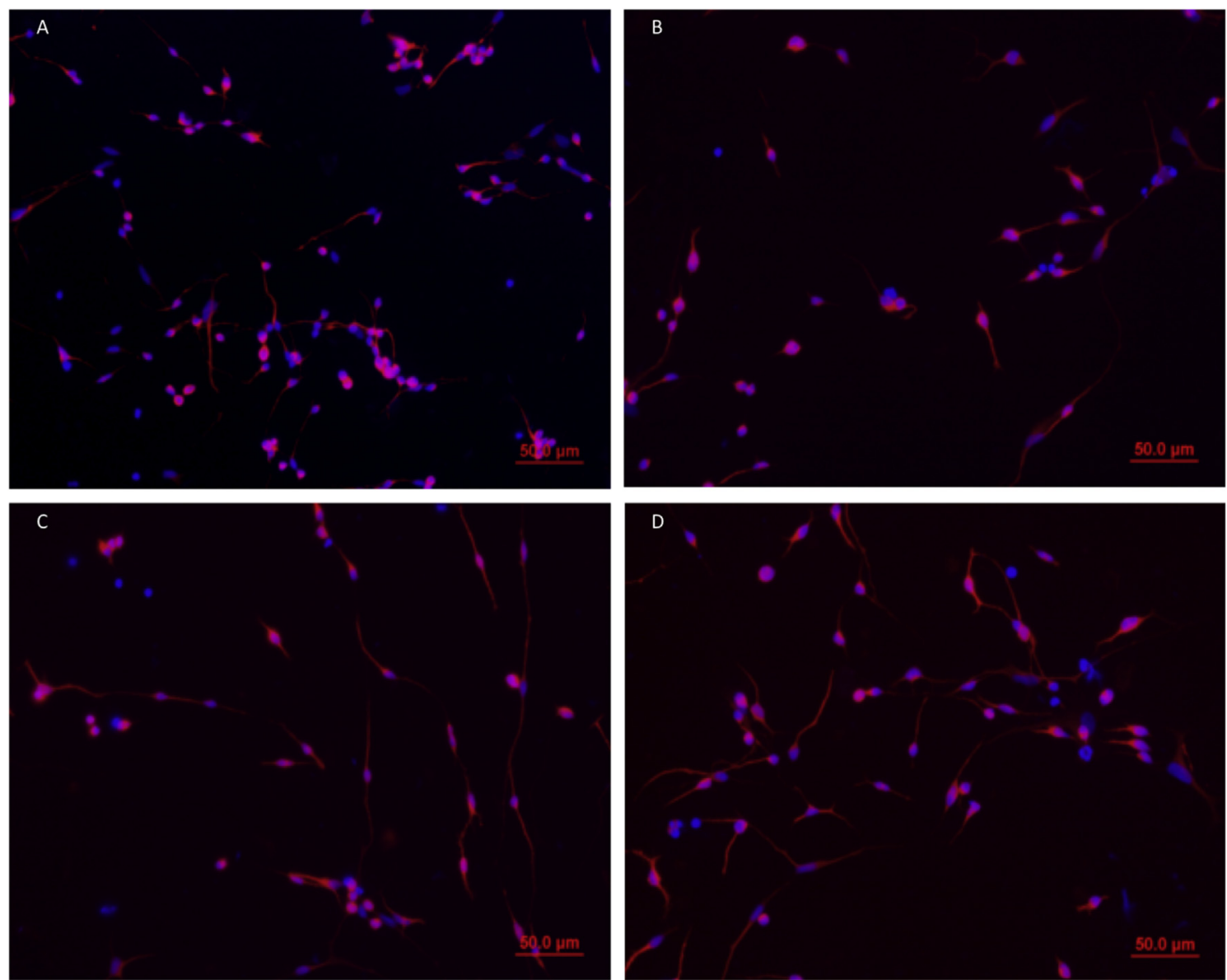

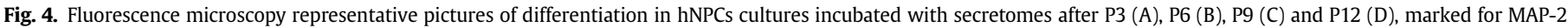
(red) and DAPI (blue).

among the cultures incubated with the different secretomes (Figs. 3 and 4).

In addition, the impact of hASCs secretomes on axonal growth, through the incubation of DRGs in different secretomes, was also evaluated. After incubation, immunocytochemistry for neurofilament was performed and the total area of neurite outgrowth was determined for each condition. Once again, no statistically significant differences were detected, when comparing all secretomes tested (Figs. 5 and 6).

In order to further understand the results obtained, a proteomic analysis of the hASCs secretomes for the above referred passages was performed, allowing the characterization of its protein profile

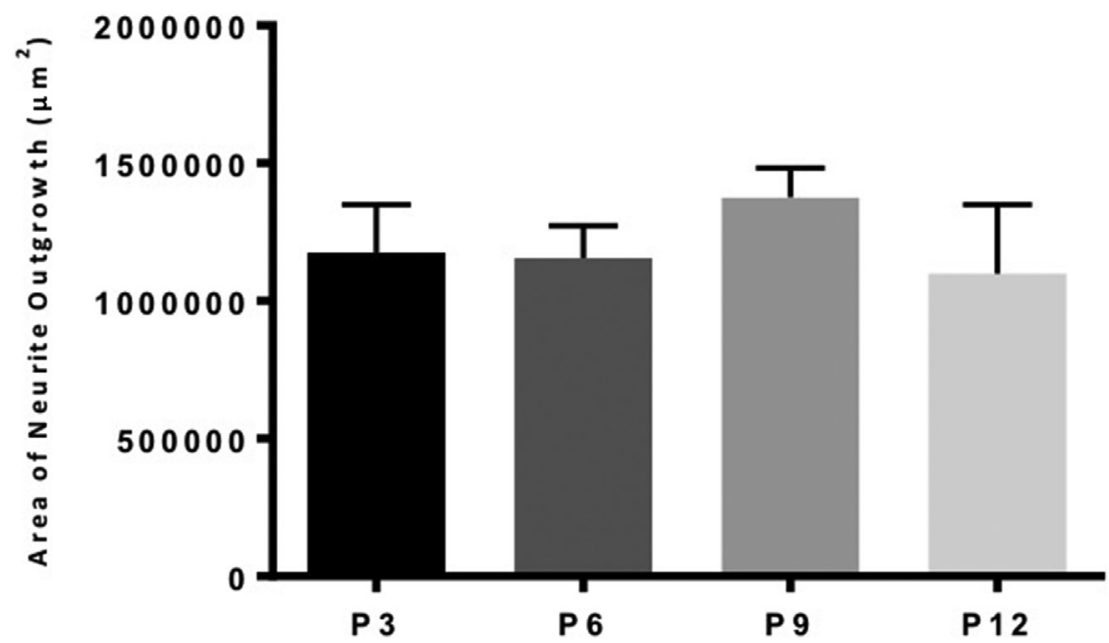

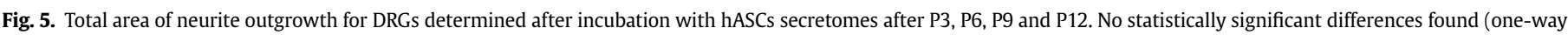
ANOVA, $n=3$, mean $\pm S D, p<0,05$ ). 

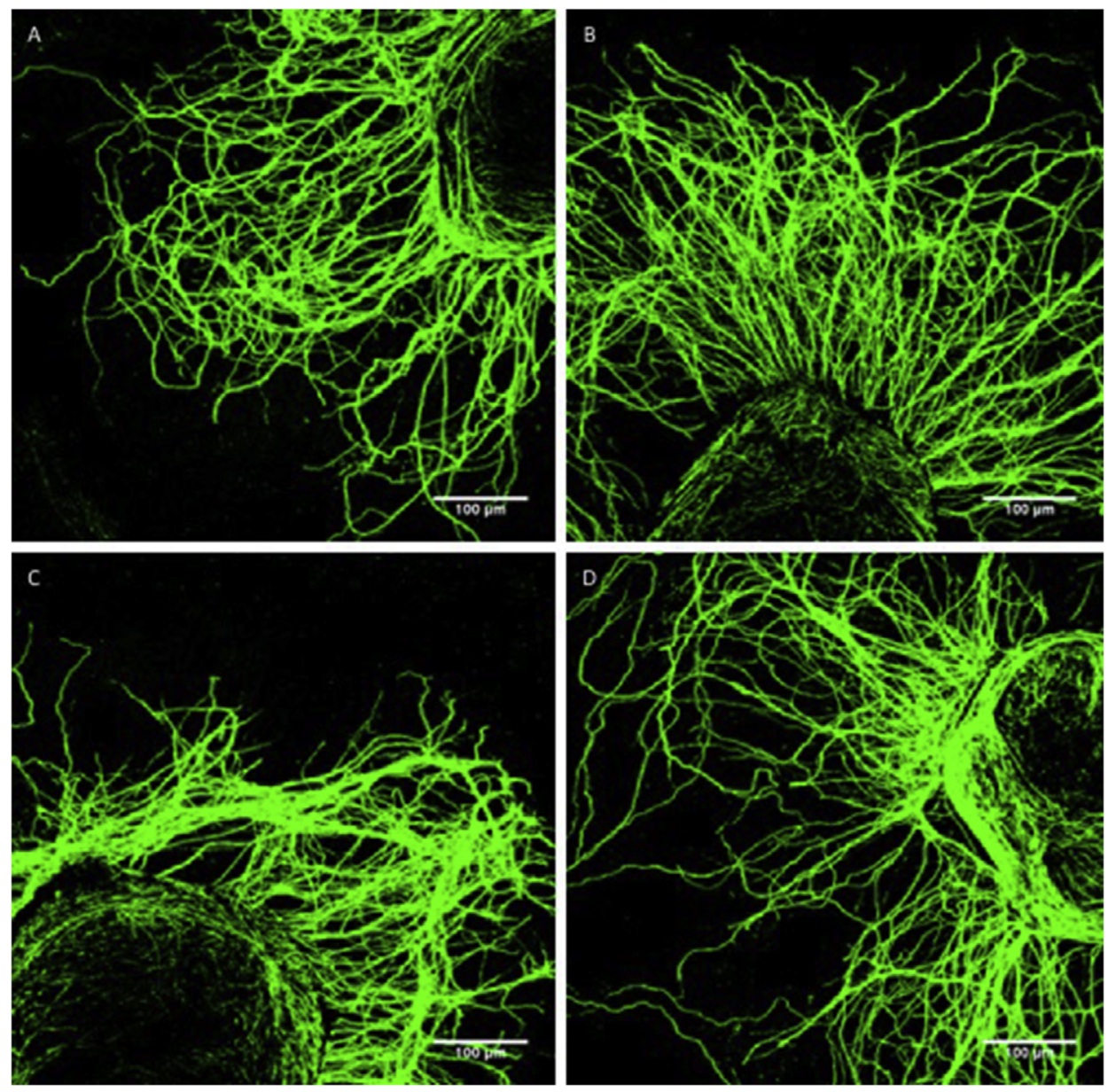

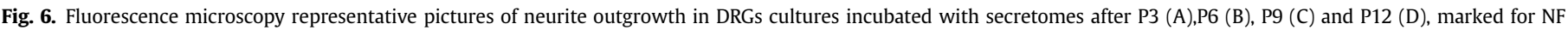
(green, i.e. marker for neurofilament).

throughout successive passaging (Fig. 7).

From the heatmap obtained it is clear that all different passages present a distinct profile. However, when specifically analyzing proteins associated to neuroprotection, neurodifferentiation and/or axonal growth (Table 1) this was not the case. Indeed, for all referred proteins, no differences were found among the $\mathrm{CM}$ collected at P3, P6, P9 and P12 (Fig. 8).

An analysis, using STRING (Search Tool for the Retrieval of Interacting Genes/Proteins; https://string-db.org/) database, was also performed to identify the processes and pathways in which the proteins presented in Table 1 could be involved (Fig. 1 - Supplementary data).

\section{Discussion}

The availability of adipose tissue as well as a straightforward isolation process, which leads to a high number of cells when compared with other sources of MSC-like cells, makes the use of hASCs attractive for CNS regenerative medicine purposes [27]. As previously discussed, their therapeutic potential has been attributed to the neuroregulatory properties of the soluble factors they secrete $[13,14,19,28]$. Indeed, previous works have reported that the secretome of ASCs increases the viability of hippocampal cultures, as well as the number of neurons in culture [14]. Moreover, it was also shown that ASCs secretome has the ability to induce neuronal differentiation of human neural progenitor cells [59]. Recently, it has been shown that exposure to ASCs secretome promotes axonal growth [29,59]. In the present study we have observed that these effects are not dependent on cell passaging. Indeed,none of the parameters referred above and here in studied, was impacted by the number of passages (P3-P12) of ASCs.

As the literature has reported, the ability of the hASCs secreted factors in promoting neuronal survival and inducing differentiation is well described. In fact, the presence of growth factors like VEGF, HGF, IGF-1, TGF- $\beta 1$ in the secretome of hASCs has been demonstrated before $[13,19]$. The proteomic analysis performed in our study identified several proteins, some of which have a neuroprotective and/or neurodifferentiation role associated as well as axonal growth promotion ability. The quantification of the latter did not reveal differences among the passages that were studied, a fact that nicely correlates with the data obtained with the cell culture experiments. Neverthles, they are a strong evidence of the key role of ASCs secretome on regulating neuronal cell viability, differentiation and axonal outgrowth.

For instance, heatshock protein 70 (Hsp70) is expressed in a ubiquitous way and it possesses a neuroprotective effect in neuronal cells [30]. In a similar way, it has also been described a possible ubiquitin carboxy-terminal hydrolase L1 (UCHL1) protein dysfunction in the pathogenesis of Parkinson and Alzheimer diseases [31]. Chiba et al. demonstrated that the neurogenic effect promoted by estrogen in the hippocampus of rats was due to their ability to induce the expression of granulin [32]. The extended presence of glia-derived nexin in the hippocampus after transient ischemia delayed neuronal death and therefore a neuroprotective 


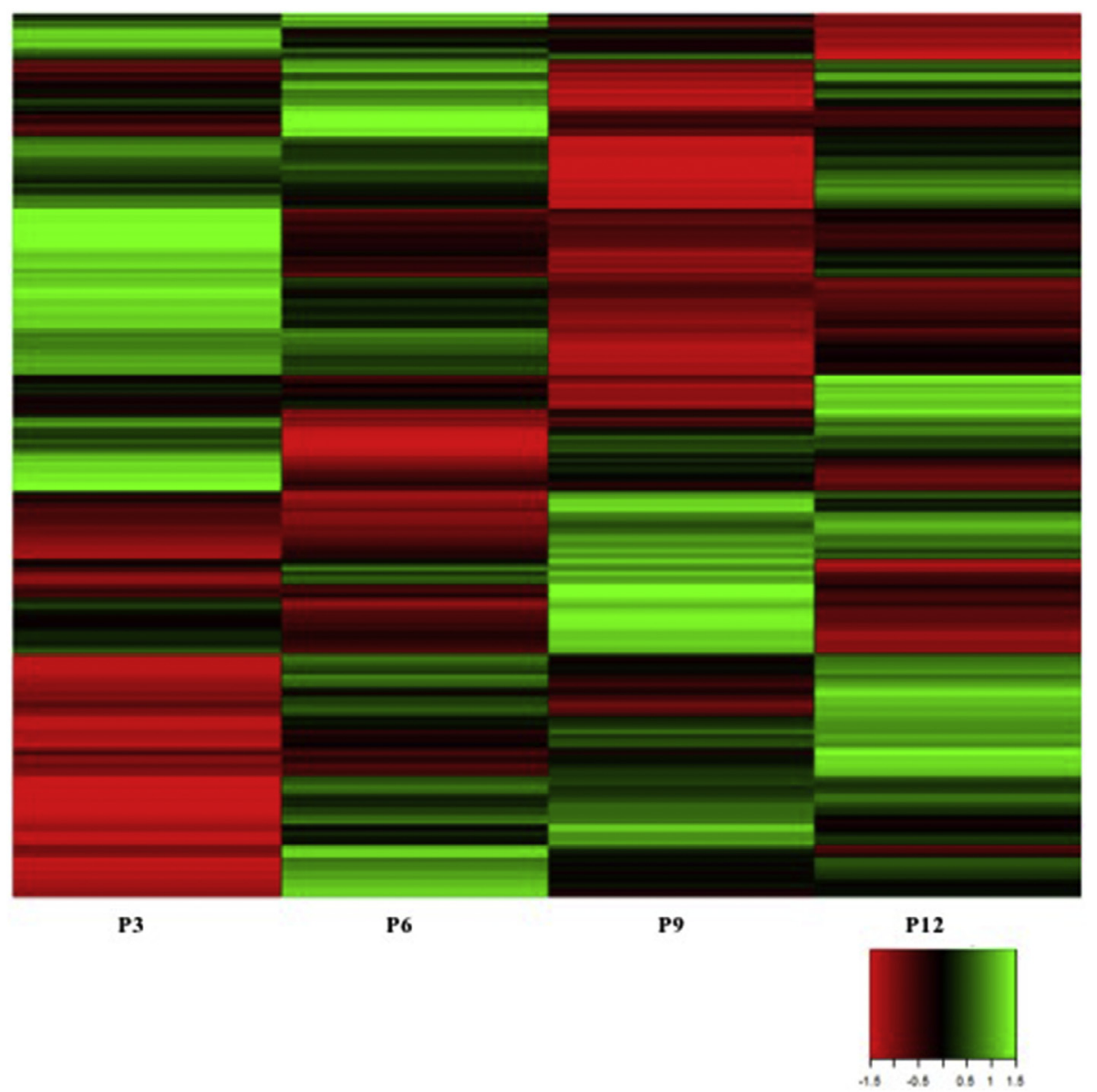

Fig. 7. Heatmap for hASCs secretomes proteomic analysis, after passages P3, P6, P9 and P12 ( $\mathrm{n}=3 /$ passage).

Table 1

List of proteins by neuronal function.

\begin{tabular}{lll}
\hline Neuroprotection & Neurodifferentiation & Axonal Growth \\
\hline Hsp70 & Thrombospondin-1 & Clusterin \\
UCHL1 & PEDF & Galectin 1 \\
Granulin & DJ-1 & Semaphorin 7A \\
Glia-derived nexin & IL-6 & Cadherin-11 \\
Thrombospondin-1 & Clusterin & Nidogen-1 \\
PEDF & Galectin 1 & Glypican-1 \\
DJ-1 & & \\
IL-6 & & \\
Clusterin & & \\
Galectin 1 & \\
\hline
\end{tabular}

effect was associated with this protein [33].

Besides the above referred proteins, some others were detected that are involved both in neuroprotection and neurodifferentiation. For example, glycoprotein thrombospondin-1 has the ability to promote the activation of TGF- $\beta 1$, a cytokine known to have a potent neurotrophic activity [34]. TGF- $\beta 1$ was shown to be related with the suppression of inflammatory processes and the promotion of neuronal survival, as well as with the promotion of sprouting and neurite outgrowth in rat hippocampal neuron cultures [35,36]. Its role in the maintenance of hNPCs and promotion of their neuronal differentiation was also already demonstrated [37].

The neuroprotective and neurodifferentiation character of other proteins found such as pigment epithelium-derived factor (PEDF), clusterin and galectin 1 has also been described in the literature
[38-42]. From these, galectin 1 stands out, as it is able to induce BDNF production by astrocytes, a growth factor known to promote neuronal survival [43].

Additionally, interleukin-6 (IL-6), a cytokine with a broad range of functions, is also present within ASCs secretome. The inhibition of apoptosis by NMDA channels was identified as the mechanism responsible for its neuroprotective effect [44]. In the same way, IL-6 derived from astrocytes has shown to promote neuronal differentiation of neuronal progenitor cells [45]. On the other hand, the exact mechanism through which DJ-1 works is still unknown, but it was already demonstrated its protective role regarding neuronal death [46]. Li et al. have reported a strong association between DJ-1 and neuronal stem cells differentiation [47].

Of the proteins mentioned, clusterin and galectin 1 also present an ability to promote axonal growth. The literature shows more than one example where clusterin has demonstrated the ability to enhance neurite outgrowth and extension [48-50]. Concerning galectin 1, it has been implicated in several processes related with axon guidance and regeneration. As reviewed by Gaudet et al. [51], it seems to be essential for the correct guidance of primary axons to specific targets and also to be involved in the regenerative response of axons after peripheral nerve injury. Quintá et al. [52] also related its presence to the promotion of axonal regeneration in a model of spinal cord injury.

Proteins related only with the promotion of axonal growth were also detected. One example is Semaphorin 7A, that has previously shown to enhance axon growth in central and peripheral nervous system and is essential for axon formation in development [53]. On 

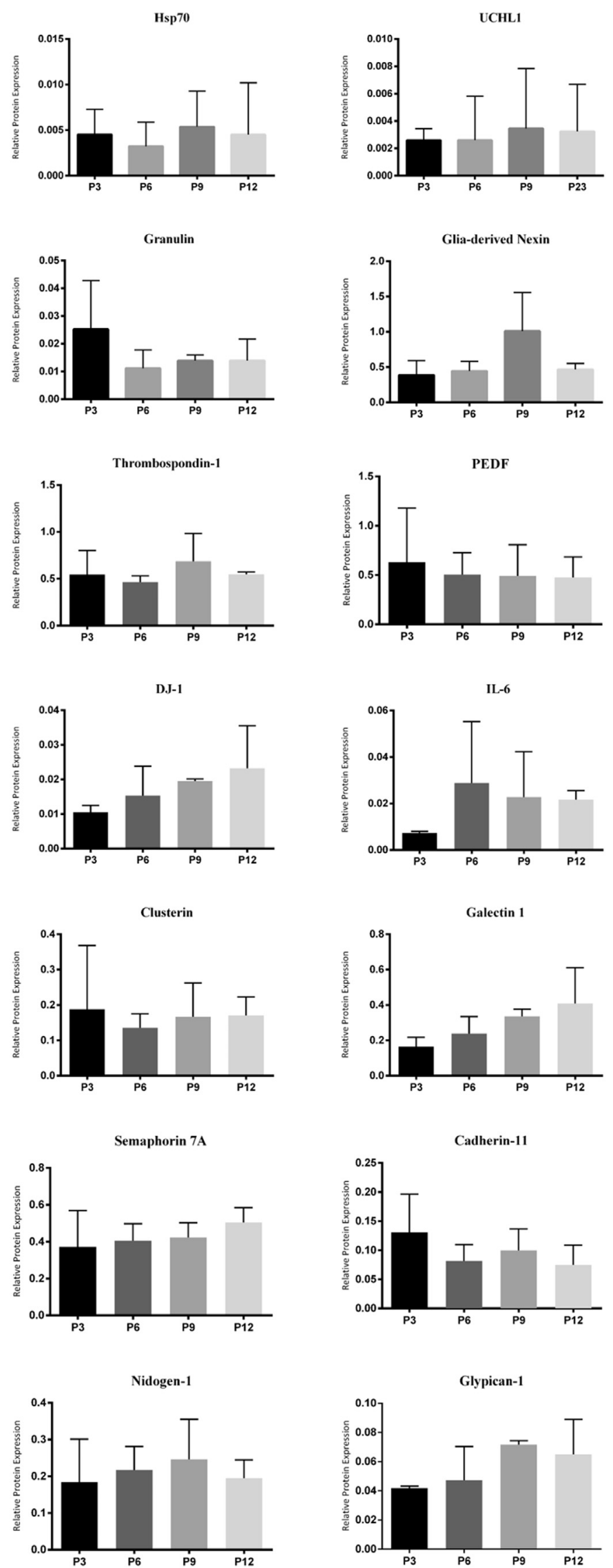

Fig. 8. Identification of proteins with neuroregulatory character within the P3, P6, P9 and P12 secretomes of ASCs. (one-way ANOVA, $n=3$, mean $\pm S D, p<0,05$ ). the other hand, Cadherin-11 has proven to promote axonal extension [54] and to be involved in the regulation of axon elongation [55], both in a model of spinal cord explants. Lee et al. [56] showed that blocking nidogen function in a model of peripheral nerve injury stopped regeneration of axons in adult sensory neurons, suggesting an essential role of this molecule in such process. Another example is glypican-1, which has more than once been implicated in the promotion of neurite outgrowth [57,58].

The STRING analysis showed that all the proteins already mentioned are involved in different pathways concerning neuroprotection, neurodifferentiation and/or axonal growth. Of these, the most relevant are some signaling pathways, namely PI3K-AKT [60-62], Wnt [63-65], mTOR [66-68], VEGF [69-71] and Notch $[72,73]$, and also other processes such as Hsp70 protein binding $[74,75]$, axonogenesis and axon guidance.

\section{Conclusions}

Our results demonstrate that successive cellular passaging does not significantly influence the neuroregulatory proteic profile of hASCs secretome and its ability to induce neurodifferentiation, post-natal neuronal survival and axonal growth. Together with the fact that adipose tissue stem cells maintain their phenotype and the properties that define them as MSCs throughout successive passaging the results obtained may allow for a longer applicability window, by possibly facilitating the cellular expansion process necessary to obtain the large numbers of cells usually needed.

To determine whether or not cellular passaging influences secretome properties is a sole characteristic of ASCs or common to all MSCs, future studies with a similar experimental design should be performed using MSCs from different sources, such as bone marrow and umbilical cord tissue.

\section{Acknowledgements}

The authors acknowledge the financial support by Prémios Santa Casa Neurociências - Prize Melo e Castro for Spinal Cord Injury Research (MC-17-2013 and MC-04-2017); Portuguese Foundation for Science and Technology through an IF Development Grant to A. J. Salgado (IF/00111/2013). Canada Research Chair in Biomedical Engineering (LAB). This work was funded by national funds through FCT under the scope of grant reference TUBITAK/ $0007 / 2014$. This article has been developed under the scope of the project NORTE-01-0145-FEDER-000013, supported by the Northern Portugal Regional Operational Programme (NORTE 2020), under the Portugal 2020 Partnership Agreement, through the European Regional Development Fund (FEDER). This work has also been funded by FEDER funds, through the Competitiveness Factors Operational Programme (COMPETE), and by National funds, through the Foundation for Science and Technology (FCT), under the scope of the projects POCI-01-0145-FEDER-007038, PTDC/NEUNMC/0205/2012, POCI-01-0145-FEDER-016428 (ref.: SAICTPAC) 0010/2015); and by The National Mass Spectrometry Network (RNEM) under the contract LISBOA-01-0145-FEDER-402-022125 (ref.: ROTEIRO/0028/2013). SIA was supported by PhD fellowship SFRH/BD/81495/2011, co-financed by the European Social Fund through the $\mathrm{POCH}$ - Programa Operacional do Capital Humano and national funds via FCT.

\section{Appendix A. Supplementary data}

Supplementary data to this article can be found online at https://doi.org/10.1016/j.biochi.2018.09.012. 


\section{References}

[1] A.J. Friedenstein, U.F. Deriglasova, N.N. Kulagina, et al., Precursors for fibroblasts in different populations of hematopoietic cells as detected by the in vitro colony assay method, Exp. Hematol. 2 (2) (1974) 83-92.

[2] D.J. Prockop, Marrow stromal cells as stem cells for nonhematopoietic tissues, Science 276 (5309) (1997) 71-74.

[3] H.K. Salem, C. Thiemermann, Mesenchymal stromal cells: current understanding and clinical status, Stem Cell. 28 (3) (2010) 585-596.

[4] P.A. Zuk, M. Zuk, P. Ashjian, et al., Human adipose tissue is a source of multipotent stem cells, Mol. Biol. Cell 13 (12) (2002) 4279-4295.

[5] P.A. Zuk, M. Zuk, H. Mizuno, et al., Multilineage cells from human adipose tissue: implications for cell-based therapies, Tissue Eng. 7 (2) (2001) 211-228.

[6] A. Erices, P. Conget, J.J. Minguell, Mesenchymal progenitor cells in human umbilical cord blood, Br. J. Haematol. 109 (1) (2000) 235-242.

[7] H.S. Wang, S.C. Hung, S.T. Peng, et al., Mesenchymal stem cells in the Wharton's jelly of the human umbilical cord, Stem Cell. 22 (7) (2004) 1330-1337.

[8] S. Wang, X. Ou, R.C. Zhao, Mesenchymal stem cells hold promise for regenerative medicine, Front. Med. 5 (4) (2011) 372-378.

[9] G. Bouchez, L. Sensebe, P. Vourc'h, et al., Partial recovery of dopaminergic pathway after graft of adult mesenchymal stem cells in a rat model of Parkinson's disease, Neurochem. Int. 52 (7) (2008) 1332-1342.

[10] M.L. Weiss, S. Medicetty, A.R. Bledsoe, et al., Human umbilical cord matrix stem cells: preliminary characterization and effect of transplantation in a rodent model of Parkinson's disease, Stem Cell. 24 (3) (2006) 781-792.

[11] D. Cizkova, J. Rosocha, I. Vanicky, et al., Transplants of human mesenchyma stem cells improve functional recovery after spinal cord injury in the rat, Cell Mol. Neurobiol. 26 (7-8) (2006) 1167-1180.

[12] H.L. Kyung, H. Suh-Kim, S.C. Ji, et al., Human mesenchymal stem cell transplantation promotes functional recovery following acute spinal cord injury in rat, Acta Neurobiol. Exp. 67 (1) (2007) 13-22.

[13] A.J. Salgado, R.L. Reis, N.J. Sousa, et al., Adipose tissue derived stem cells secretome: soluble factors and their roles in regenerative medicine, Curr. Stem Cell Res. Ther. 5 (2) (2010) 103-110.

[14] C.A. Ribeiro, J.S. Fraga, M. Graos, et al., The secretome of stem cells isolated from the adipose tissue and Wharton jelly acts differently on central nervous system derived cell populations, Stem Cell Res. Ther. 3 (3) (2012) 18.

[15] A.J. Salgado, J.S. Fraga, A.R. Mesquita, et al., Role of human umbilical cord mesenchymal progenitors conditioned media in neuronal/glial cell densities, viability, and proliferation, Stem Cell. Dev. 19 (7) (2010) 1067-1074.

[16] A.I. Caplan, J.E. Dennis, Mesenchymal stem cells as trophic mediators, J. Cell. Biochem. 98 (5) (2006) 1076-1084.

[17] A. Wilkins, K. Kemp, M. Ginty, et al., Human bone marrow-derived mesenchymal stem cells secrete brain-derived neurotrophic factor which promotes neuronal survival in vitro, Stem Cell Res. 3 (1) (2009) 63-70.

[18] C.A. Ribeiro, A.J. Salgado, J.S. Fraga, et al., The secretome of bone marrow mesenchymal stem cells - conditioned media varies with time and drives a distinct effect on mature neurons and glial cells (primary cultures), Journal of Tissue Engeneering and Regenerative Medicine 5 (8) (2011) 668-672.

[19] J. Rehman, D. Traktuev, J. Li, et al., Secretion of angiogenic and antiapoptotic factors by human adipose stromal cells, Circulation 109 (10) (2004) 1292-1298.

[20] S.H. Koh, K.S. Kim, M.R. Choi, et al, Implantation of human umbilical cordderived mesenchymal stem cells as a neuroprotective therapy for ischemic stroke in rats, Brain Res. 1229 (2008) 233-248.

[21] B.A. Baghbaderani, K. Mukhida, A. Sen, et al., Bioreactor expansion of human neural precursor cells in serum-free media retains neurogenic potential, Biotechnol. Bioeng. 105 (2010) 823-833.

[22] I. Allodi, M.S. Guzmán-Lenis, J. Hernàndez, et al., In vitro comparison of motor and sensory neuron outgrowth in a 3D collagen matrix, J. Neurosci. Methods 198 (2011) 53-61.

[23] F.G. Teixeira, K.M. Panchalingam, S.I. Anjo, et al., Do hypoxia/normoxia culturing conditions change the neuroregulatory profile of Wharton jelly mesenchymal stem cell secretome? Stem Cell Res. Ther. 6 (2015) 133-147.

[24] B.J. Manadas, K. Vougas, M. Fountoulakis, et al., Sample sonication after trichloroacetic acid precipitation increases protein recovery from cultured hippocampal neurons, and improves resolution and reproducibility in twodimensional gel electrophoresis, Electrophoresis 27 (2006) 1825-1831.

[25] S.I. Anjo, C. Santa, B. Manadas, Short GeLCSWATH: a fast and reliable quantitative approach for proteomic screenings, Proteomics 15 (2015) 757-762.

[26] B.C. Collins, L.C. Gillet, G. Rosenberger, et al., Quantifying protein interaction dynamics by SWATH mass spectrometry: application to the 14-3-3 system, Nat. Methods 10 (2013) 1246-1253.

[27] A. Schäffler, C. Büchler, Adipose tissue-derived stromal cells - basic and clinical applications for novel cell therapies, Stem Cell. 25 (4) (2007) 818-827.

[28] F.G. Teixeira, M.M. Carvalho, A. Neves-Carvalho, et al., Secretome of mesenchymal progenitors from the umbilical cord acts as modulator of neural/glial proliferation and differentiation, Stem Cell Rev. 11 (2) (2015) 288-297.

[29] E.D. Gomes, S.S. Mendes, R.C. Assunção-Silva, et al., Co-transplantation of adipose tissue-derived stromal cells and olfactory ensheathing cells for spinal cord injury repair, Stem Cell. 36 (5) (2018) 696-708.

[30] H.P. Bonner, C.G. Concannon, C. Bonner, et al., Differential expression patterns of Puma and Hsp70 following proteasomal stress in the hippocampus are key determinants of neuronal vulnerability, J. Neurochem. 114 (2) (2010)
606-616.

[31] S. Roche, G. D'Ippolito, L.A. Gomez, et al., Comparative analysis of protein expression of three stem cell populations: models of cytokine delivery system in vivo, Int. J. Pharmacol. 40 (1) (2013) 72-82.

[32] S. Chiba, M. Suzuki, K. Yamanouchi, et al., Involvement of granulin in estrogen-induced neurogenesis in the adult rat hippocampus, J. Reprod. Dev. 53 (2) (2007) 297-307.

[33] M.C. Hoffmann, C. Nitsch, A.L. Scotti, et al., The prolonged presence of gliaderived nexin, an endogenous protease inhibitor, in the hippocampus after ischemia-induced delayed neuronal death, Neuroscience 49 (2) (1992) 397-408.

[34] S.E. Crawford, V. Stellmach, J.E. Murphy-Ullrich, et al., Thrombospondin-1 is a major activator of TGF- beta1 in vivo, Cell 93 (7) (1998) 1159-1170.

[35] M. Makwana, L.L. Jones, D. Cuthill, et al., Endogenous transforming growth factor beta 1 suppresses inflammation and promotes survival in adult CNS, J. Neurosci. 27 (42) (2007) 11201-11213.

[36] A. Ishihara, H. Saito, K. Abe, Transforming growth factor-beta 1 and -beta 2 promote neurite sprouting and elongation of cultured rat hippocampal neurons, Brain Res. 639 (1) (1994) 21-25.

[37] Z. Lu, J. Kipnis, Thrombospondin 1 - a key astrocyte-derived neurogenic factor FASEB (Fed. Am. Soc. Exp. Biol.) J. 24 (6) (2010) 1925-1934.

[38] A. Sanchez, D. Tripathy, X. Yin, et al., Pigment epithelium-derived factor (PEDF) protects cortical neurons in vitro from oxidant injury by activation of extracellular signal-regulated kinase (ERK) $1 / 2$ and induction of Bcl-2, Neurosci. Res. 72 (1) (2012) 1-8.

[39] J. Tombran-Tink, G.G. Chader, L.V. Johnson, PEDF: a pigment epitheliumderived factor with potent neuronal differentiative activity, Exp. Eye Res. 53 (3) (1991) 411-414.

[40] G. Wicher, A. Fex-Svenningsen, Velsecchi, et al., Extracellular clusterin promotes neuronal network complexity in vitro, Neuroreport 19 (15) (2008) $1487-1491$.

[41] O. Cordero-Llana, S.A. Scott, S.L. Maslen, et al., Clusterin secreted by astrocytes enhances neuronal differentiation from human neural precursor cells, Cell Death Differ. 18 (5) (2011) 907-913.

[42] M. Sakaguchi, T. Shingo, T. Shimazaki, et al., A carbohydrate-binding protein, Galectin-1, promotes proliferation of adult neural stem cells, Proceedings of the National Academy of Sciences of the USA 103 (18) (2006) 7112-7117.

[43] W.S. Qu, Y.H. Wang, J.P. Wang, et al., Galectin-1 enhances astrocytic BDNF production and improves functional outcome in rats following ischemia, Neurochem. Res. 35 (11) (2010) 1716-1724.

[44] Z. Liu, Y.H. Qiu, B. Li, et al., Neuroprotection of interleukin-6 against NMDAinduced apoptosis and its signal-transduction mechanisms, Neurotox. Res. 19 (3) (2011) 484-495.

[45] J. Oh, M.A. McCloskey, C.C. Blong, et al., Astrocyte-derived interleukin-6 promotes specific neuronal differentiation of neural progenitor cells from adult hippocampus, J. Neurosci. Res. 88 (13) (2010) 2798-2809.

[46] R.M. Canet-Avilés, M.A. Wilson, D.W. Miller, et al., The Parkinson's disease protein DJ-1 is neuroprotective due to cysteine-sulfinic acid-driven mitochondrial localization, Proceedings of the National Academy of Sciences of the USA 101 (24) (2004) 9103-9108.

[47] S. Li, Y. Sun, X. Zhao, et al., Expression of the Parkinson's disease protein DJ-1 during the differentiation of neural stem cells, Brain Res. 1468 (2012) 84-93.

[48] S.W. Kang, Y.J. Shin, Y.J. Shim, et al., Clusterin interacts with SCLIP (SCG10-like protein) and promotes neurite outgrowth of PC12 cells, Exp. Cell Res. 309 (2) (2005) 305-315.

[49] F. White, J.A. Nicoll, K. Horsburgh, Alterations in ApoE and ApoJ in relation to degeneration and regeneration in a mouse model of entorhinal cortex lesion, Exp. Neurol. 169 (2) (2001) 307-318.

[50] G. Wicher, A. Fex-Svenningsen, I. Velsecchi, et al., Extracellular clusterin promotes neuronal network complexity in vitro, Neuroreport 19 (15) (2008) 1487-1491.

[51] A.D. Gaudet, J.D. Steeves, W. Tetzlaff, et al., Expression and functions of galectin-1 in sensory and motoneurons, Curr. Drug Targets 6 (4) (2005) 419-425.

[52] H.R. Quintá, J.M. Pasquini, G.A. Rabinovich, et al., Glycan-dependent binding of galectin-1 to neuropilin-1 promotes axonal regeneration after spinal cord injury, Cell Death Differ. 21 (6) (2014) 941-955.

[53] R.J. Pasterkamp, J.J. Peschon, M.K. Spriggs, et al., Semaphorin 7A promotes axon outgrowth through integrins and MAPKs, Nature 424 (2003) 398-405.

[54] C. Boscher, R.M. Mège, Cadherin-11 interacts with the FGF receptor and induces neurite outgrowth through associated downstream signalling, Cell. Signal. 20 (6) (2008) 1061-1072.

[55] V. Marthiens, J. Gavard, F. Padilla, et al., A novel function for cadherin-11 in the regulation of motor axon elongation and fasciculation, Mol. Cell. Neurosci. 28 (4) (2005) 715-726.

[56] H.K. Lee, I.A. Seo, D.J. Suh, et al., Nidogen plays a role in the regenerative axon growth of adult sensory neurons through Schwann cells, J. Kor. Med. Sci. 24 (4) (2009) 654-659.

[57] K. Akita, M. Toda, Y. Hosoki, et al., Heparan sulphate proteoglycans interact with neurocan and promote neurite outgrowth from cerebellar granule cells, Biochem. J. 383 (Pt 1) (2004) 129-138.

[58] J.J. Hill, K. Jin, X.O. Mao, et al., Intracerebral chondroitinase ABC and heparan sulfate proteoglycan glypican improve outcome from chronic stroke in rats, Proc. Natl. Acad. Sci. U.S.A. 109 (23) (2012) 9155-9160

[59] R.C. Assunção-Silva, B. Mendes-Pinheiro, P. Patrício, et al., Exlpoiting the 
impact of the secretome of mscs isolated from diferente sources on neuronal differentiation and axonal growth, Biochimie (2018), https://doi.org/10.1016/ j.biochi.2018.07.026 in press.

[60] H. Wang, S. Liao, R. Geng, et al., IGF-1 signaling via the PI3K/Akt pathway confers neuroprotection in human retinal pigment epithelial cells exposed to sodium nitroprusside insult, J. Mol. Neurosci. 55 (4) (2015) 931-940.

[61] L.M. Shen, Z.W. Song, Y. Hua, et al., miR-181d-5p promotes neurite outgrowth in PC12 Cells via PI3K/Akt pathway, CNS Neurosci. Ther. 23 (11) (2017) 894-906.

[62] X. Zhang, L. Zhang, X. Cheng, et al., IGF-1 promotes Brn-4 expression and neuronal differentiation of neural stem cells via the PI3K/Akt pathway, PloS One 9 (12) (2014), e113801.

[63] B. Marchetti, F. L'Episcopo, M.C. Morale, et al., Uncovering novel actors in astrocyte-neuron crosstalk in Parkinson's disease: the Wnt/ $\beta$-catenin signaling cascade as the common final pathway for neuroprotection and selfrepair, Eur. J. Neurosci. 37 (10) (2013) 1550-1563.

[64] L. Wang, Y. Liu, S. Li, et al., Wnt signaling pathway participates in valproic acid-induced neuronal differentiation of neural stem cells, Int. J. Clin. Exp. Pathol. 8 (1) (2015) 578-585.

[65] A.L. Garcia, A. Udeh, K. Kalahasty, et al., A growing field: the regulation of axonal regeneration by Wnt signaling, Neural Regen Res 13 (1) (2018) 43-52.

[66] H. Kanno, H. Ozawa, A. Sekiguchi, et al., The role of mTOR signaling pathway in spinal cord injury, Cell Cycle 11 (17) (2012) 3175-3179.

[67] J.M. Bateman, Mechanistic insights into the role of mTOR signaling in neuronal differentiation, Neurogenesis (Austin) 2 (1) (2015), e1058684.

[68] J. Bockaert, P. Marin, mTOR in brain physiology and pathologies, Physiol. Rev. 95 (4) (2015) 1157-1187.

[69] T. Hao, P. Rockwell, Signaling through the vascular endothelial growth factor receptor VEGFR-2 protects hippocampal neurons from mitochondrial dysfunction and oxidative stress, Free Radic. Biol. Med. 63 (2013) 421-431.

[70] Y. Li, J. Luo, W.M. Lau, et al., Caveolin-1 plays a crucial role in inhibiting neuronal differentiation of neural stem/progenitor cells via VEGF signalingdependent pathway, PloS One 6 (8) (2011), e22901.

[71] M. Dumpich, H.G. Mannherz, C. Theiss, VEGF signaling regulates cofilin and the Arp2/3-complex within the axonal growth cone, Curr. Neurovascular Res. 12 (3) (2015) 293-307.

[72] Z. Li, J. Wang, C. Zhao, et al., Acute blockage of Notch signaling by DAPT induces neuroprotection and neurogenesis in the neonatal rat brain after stroke, Transl Stroke Res 7 (2) (2016) 132-140.

[73] Y. Wei, G.N. Pandian, Z. Yu, et al., Synthetic DNA-binding inhibitor of HES1 alters the Notch signaling pathway and induces neuronal differentiation, ACS Omega 3 (3) (2018) 3608-3616.

[74] B. Sabirzhanov, B.A. Stoica, M. Hanscom, et al., Over-expression of HSP70 attenuates caspase-dependent and caspase-independent pathways and inhibits neuronal apoptosis, J. Neurochem. 123 (4) (2012) 542-554.

[75] K. Frebel, S. Wiese, N. Funk, et al., Differential modulation of neurite growth by the S- and the L-forms of bag1, a co-chaperone of Hsp70, Neurodegener. Dis. 4 $(2-3)(2007)$ 261-269. 